\title{
Vigilância de ambientes da febre maculosa: explorando as áreas silenciosas do Brasil
}

Surveillance of environments of spotted fever: exploring the quiet areas of Brazil

Vigilancia de ambientes de la fiebre maculosa: explorando las áreas silenciosas de Brasil

Stefan Vilges de Oliveira

Unidade Técnica de Vigilância de Zoonoses, Coordenação Geral de Doenças Transmissíveis, Departamento de Vigilância Epidemiológica, Secretaria de Vigilância em Saúde, Ministério da Saúde, Brasília, Distrito Federal, Brasil

Programa de Pós-Graduação em Medicina Tropical, Universidade de Brasília, Brasília, Distrito Federal, Brasil

\section{Simone Valeria Costa Pereira}

Unidade Técnica de Vigilância de Zoonoses, Coordenação Geral de Doenças Transmissíveis, Departamento de Vigilância Epidemiológica, Secretaria de Vigilância em Saúde, Ministério da Saúde, Brasília, Distrito Federal, Brasil

Fernanda Voietta Pinna

Unidade Técnica de Vigilância de Zoonoses, Coordenação Geral de Doenças Transmissíveis, Departamento de Vigilância Epidemiológica, Secretaria de Vigilância em Saúde, Ministério da Saúde, Brasília, Distrito Federal, Brasil

\section{Lidsy Ximenes Fonseca}

Unidade Técnica de Vigilância de Zoonoses, Coordenação Geral de Doenças Transmissíveis, Departamento de Vigilância Epidemiológica, Secretaria de Vigilância em Saúde, Ministério da Saúde, Brasília, Distrito Federal, Brasil
Nicolau Mavés da Serra-Freire

Laboratório de Referência Nacional em Vetores das Riquetsioses, Instituto Oswaldo Cruz/Fiocruz, Rio de Janeiro, Rio de Janeiro, Brasil

Karen Medeiros Cardoso

Laboratório de Referência Nacional em Vetores das Riquetsioses, Instituto Oswaldo Cruz/Fiocruz, Rio de Janeiro, Rio de Janeiro, Brasil

Ana Beatriz Pais Borsoi

Laboratório de Referência Nacional em Vetores das Riquetsioses, Instituto Oswaldo Cruz/Fiocruz, Rio de Janeiro, Rio de Janeiro, Brasil

\section{Marinete Amorim}

Laboratório de Referência Nacional em Vetores das Riquetsioses, Instituto Oswaldo Cruz/Fiocruz, Rio de Janeiro, Rio de Janeiro, Brasil

Eduardo Pacheco de Caldas

Unidade Técnica de Vigilância de Zoonoses, Coordenação Geral de Doenças Transmissíveis, Departamento de Vigilância Epidemiológica, Secretaria de Vigilância em Saúde, Ministério da Saúde, Brasília, Distrito Federal, Brasil

Gilberto Salles Gazeta

Laboratório de Referência Nacional em Vetores das Riquetsioses, Instituto Oswaldo Cruz/Fiocruz, Rio de Janeiro, Rio de Janeiro, Brasil

\section{RESUMO}

O presente estudo descreveu as intervenções realizadas em áreas silenciosas do Brasil, sob o aspecto da promoção da saúde. As áreas avaliadas durante o período de 2014 a 2015 compreenderam as seguintes Unidades Federadas: Mato Grosso do Sul, Alagoas, Pernambuco, Rondônia, Roraima, Rio Grande do Norte e Maranhão. Foram avaliados os dados sobre a vigilância da febre maculosa por meio das fichas epidemiológicas disponíveis no Sistema de Informação de Agravo de Notificação, bem como a fauna de vetores e das infecções por riquétsias do grupo da febre maculosa (RGFM). Foram coletados 1.528 vetores de dez espécies diferentes, cuja maior diversidade foi observada em Rondônia. Neste estudo, 85\% dos Estados investigados apresentaram RGFM em vetores ou em humanos, sendo Mato Grosso do Sul, Rondônia e Pernambuco, os mais prevalentes. Os resultados apresentados apontam para a necessidade permanente de qualificação das equipes de profissionais de saúde e ambiente, a fim de aprimorar os dados sobre a febre maculosa em nosso meio.

Palavras-chave: Doenças Transmitidas por Carrapatos; Zoonoses; Febre Maculosa; Capacitação em Serviço; Vigilância Sentinela.

\author{
Correspondência / Correspondence / Correspondencia: \\ Stefan Vilges de Oliveira \\ Unidade Técnica de Vigilância de Zoonoses, Coordenação Geral \\ de Doenças Transmissíveis, Departamento de Vigilância das Doenças \\ Transmissíveis, Secretaria de Vigilância em Saúde do Ministério \\ da Saúde \\ Setor Comercial Sul (SCS), quadra 04, bloco A, Edifício Principal, \\ $3^{\circ}$ andar. Bairro: Asa Sul \\ CEP: 70304-000 Brasília-Distrito Federal-Brasil \\ Tel.: +55 (61) 3213-8232 \\ E-mail: stefan.oliveira@saude.gov.br
}




\section{INTRODUÇÃO}

A febre maculosa é uma doença febril aguda causada por riquétsias, bactérias intracelulares obrigatórias, transmitidas principalmente por carrapatos'. No Brasil, Rickettsia rickettsii é a espécie mais relevante, apresentando casos graves na Região Sudeste ${ }^{2,3}$ e, mais recentemente, na Região Sul ${ }^{3}$. A doença pode evoluir de forma rápida, com um comprometimento clínico importante, apresentando elevado coeficiente de letalidade, sendo, por isso, agravo de notificação compulsória ${ }^{4}$. Amblyomma sculptum e Amblyomma aureolatum são incriminados em sua transmissão ${ }^{3}$.

Entretanto, casos mais brandos de febre maculosa foram identificados nas Regiões Sul, Sudeste e Nordeste do País $5,6,7$, apresentando linfadenopatia e escara de inoculação como manifestações clínicas relevantes. A doença pode estar associada à Rickettsia sp. cepa Mata Atlântica, espécie filogeneticamente próxima a Rickettsia parkerib,7 e tendo como vetor envolvido no ciclo epidêmico Amblyomma ovale $3,5,8,9$.

Em uma abordagem de investigação dos ambientes com potencial risco para a presença da febre maculosa, o Ministério da Saúde promoveu capacitações técnicas aos profissionais da rede do Sistema Único de Saúde, priorizando inicialmente as áreas endêmicas da doença ${ }^{10}$. Paralelamente aos treinamentos, materiais instrucionais no formato de videoaulas foram produzidos e disponibilizados, abordando os aspectos da vigilância epidemiológica e ambiental ${ }^{10,11,12}$. Essa iniciativa é parte de um processo que tem como objetivo qualificar e habilitar equipes para realizar investigações de ambientes, e essas ações buscam apoiar os programas municipais e estaduais de vigilância das doenças transmitidas por carrapatos ${ }^{10}$.

Contudo, tem-se observado baixa frequência de suspeição clínica e epidemiológica de casos na Região Norte e parte das Regiões Nordeste e Centro-Oeste, consideradas áreas silenciosas para a doença $a^{4,10,13}$. Nessas áreas o conhecimento sobre a história natural e da distribuição de riquetsioses de interesse médico ainda é incipiente? ${ }^{2}$.

O presente estudo descreveu um conjunto de iniciativas promovidas pelo Ministério da Saúde a fim de intensificar a vigilância ambiental e epidemiológica, bem como aprimorar o conhecimento sobre as áreas consideradas silenciosas para a febre maculosa no Brasil.

\section{MATERIAIS E MÉTODOS}

O estudo foi realizado de 2014 a 2015 e iniciado com o convite a todos os Estados do Brasil que, até 2013, não haviam informado casos confirmados de febre maculosa. Os Estados do Mato Grosso do Sul (MS), na Região Centro-Oeste; Alagoas $(\mathrm{AL})$, Pernambuco (PE), Rio Grande do Norte (RN) e Maranhão (MA), na Região Nordeste; e Rondônia (RO) e Roraima (RR), na Região Norte, foram incorporados ao projeto, após manifestação de interesse.

\section{CAPACITAÇÕES EM ÁREAS SILENCIOSAS}

Foram realizadas capacitações técnicas em investigações de casos e vigilância de ambientes da febre maculosa em áreas consideradas silenciosas para presença da doença. $O$ programa buscou tratar da epidemiologia das doenças transmitidas por carrapatos, das rotinas de encaminhamento de materiais biológicos para análise laboratorial, das atividades práticas de campo e de laboratório e do uso adequado de equipamentos de proteção individual e coletiva ${ }^{10,11,12}$.

\section{INVESTIGAÇÃO DA FAUNA DE VETORES E DE HOSPEDEIROS}

Foram realizadas coletas de carrapatos no ambiente e em hospedeiros. Os carrapatos foram coletados com auxílio da técnica de arrasto, armadilha de $\mathrm{CO}_{2}$ ou pela busca ativa, diretamente nos hospedeiros e no ambiente ${ }^{12}$. A prioridade foi dada para investigar as áreas urbanas e periféricas, onde havia o conhecimento prévio da densidade de vetores com consequente parasitismo humano. Essas áreas incluíram áreas públicas ou privadas, como parques, canis, haras, fazendas de universidades, abrigos de animais domésticos e silvestres ${ }^{12}$.

As amostras coletadas seguiram o fluxo da Rede Nacional de Vigilância de Ambiente para Febre Maculosa e Outras Riquetsioses, implementada pelo Ministério da Saúde durante as capacitações na investigação de casos e vigilância de ambiente para febre maculosa das áreas com casos confirmados ${ }^{10,11,12}$. Os carrapatos foram analisados e identificados de acordo com as chaves dicotômicas ${ }^{14}$.

$\mathrm{Na}$ investigação molecular dos bioagentes transmitidos por carrapatos, os vetores foram submetidos à extração de DNA e quantificação de DNA extraído, individualmente ou em pool. Foram utilizados primers gênero-específicos $(g \mid t A)$ para identificação de Rickettsia spp. e primers grupo-específico, para identificação de riquétsias do grupo da febre maculosa (RGFM) (ompA) ${ }^{15,16}$. As análises foram realizadas no Laboratório de Referência Nacional para Vetores de Riquetsioses na Fundação Oswaldo Cruz no Estado do Rio de Janeiro.

\section{ANÁLISE DE DADOS EPIDEMIOLÓGICOS}

Dados epidemiológicos dos respectivos Estados foram analisados utilizando, como base, os registros das fichas de notificação e investigação de febre maculosa inseridas no Sistema de Informação de Agravo de Notificação durante o período de 2014 a 2015. Foram considerados como caso suspeito: indivíduo que apresentou febre, cefaleia, mialgia e história de picada de carrapatos e/ou contato com animais domésticos e/ou silvestres e/ou frequentou área sabidamente de transmissão de febre maculosa nos últimos 15 dias e/ou apresente exantema maculopapular ou manifestações hemorrágicas ${ }^{11}$. E como caso confirmado: indivíduo suspeito com diagnóstico laboratorial, sorológico ou molecular confirmatório"1. Registros que não se enquadraram na definição de caso, proposta pelo MS, foram excluídos da análise ${ }^{11}$. As variáveis foram 
analisadas de acordo com a classificação do município de notificação (local onde foi notificado o caso suspeito) e do município da infecção (local onde foi comprovada, por meio de investigação epidemiológica, a autoctonia do caso ao município) $)^{4,13}$. Os dados foram apresentados por números brutos e frequências. Foram utilizados os softwares TabWin 32 v3.6b para tabulação de dados; Microsoft Office Excel 2010 para produção de planilhas e gráficos; e TerraView v3.2.0.1 para elaboração de mapas $^{4,13}$.

\section{ASPECTOS ÉTICOS}

Por se tratar de uma estratégia do serviço de vigilância em saúde, o presente estudo não foi submetido previamente a um comitê de ética em pesquisa. No entanto, obedeceu aos preceitos éticos estabelecidos na Resolução n 466/2012 do Conselho Nacional de Saúde, não abrangendo dados que pudessem identificar os indivíduos registrados no sistema de informação. A coleta de vetores em animais silvestres foi autorizada pela licença do Instituto Brasileiro de Meio Ambiente, processo $n^{\circ}$ 02001.000812/2013-01 $n^{\circ}$ 003. A contenção e manuseio de animais domésticos seguiram as normas estabelecidas pelo Conselho Federal de Medicina Veterinária.

\section{RESULTADOS}

\section{CAPACITAÇÕES EM ÁREAS SILENCIOSAS}

Foram capacitados 161 profissionais: $M S=29$; $\mathrm{AL}=18 ; \mathrm{PE}=10 ; \mathrm{RO}=18 ; \mathrm{RR}=19 ; \mathrm{RN}=29 ;$ e $M A=38$. As sete capacitações realizadas tiveram representantes de 56 municípios (Figura 1). Quanto ao perfil de escolaridade dos treinandos, 64,4\% possuíam nível superior, sendo que $7,6 \%$ tinham mestrado e 0,8\% doutorado; 22,7\% possuíam nível médio, 2,3\% nível fundamental e 2,3\% não informaram seu nível de escolaridade. Quanto à formação profissional, $28,79 \%$ eram biólogos, 21,97\% veterinários, $21,97 \%$ tinham outras formações superiores ou técnicas e $27,27 \%$ não informaram sua formação acadêmica.

Quanto à ocupação/função, 52,42\% eram responsáveis técnicos, $20,16 \%$ eram técnicos em entomologia, $12,90 \%$ eram agentes de controle de endemias e de vigilância em saúde, 8,87\% eram agentes em saúde pública, 2,42\% eram técnicos em enfermagem e 3,23\% eram estagiários, estudantes, sanitaristas ou técnicos em saneamento.

\section{INVESTIGAÇÕES DA FAUNA DE VETORES E DE HOSPEDEIROS}

Foram coletados 1.528 potenciais vetores nas áreas investigadas em múltiplos hospedeiros e ambientes (Tabela 1). A positividade para RGFM foi observada em PE, RO, RR, RN e MA (Figura 2). $\bigcirc$ Estado de Alagoas foi capacitado, mas não enviou amostras dentro do fluxo da Rede Nacional de Vigilância de Ambiente para Febre Maculosa e Outras Riquetsioses e, desta forma, não foi possível analisar a fauna de vetores desse Estado.

Unidades Federadas capacitadas na $1^{a}$ etapa

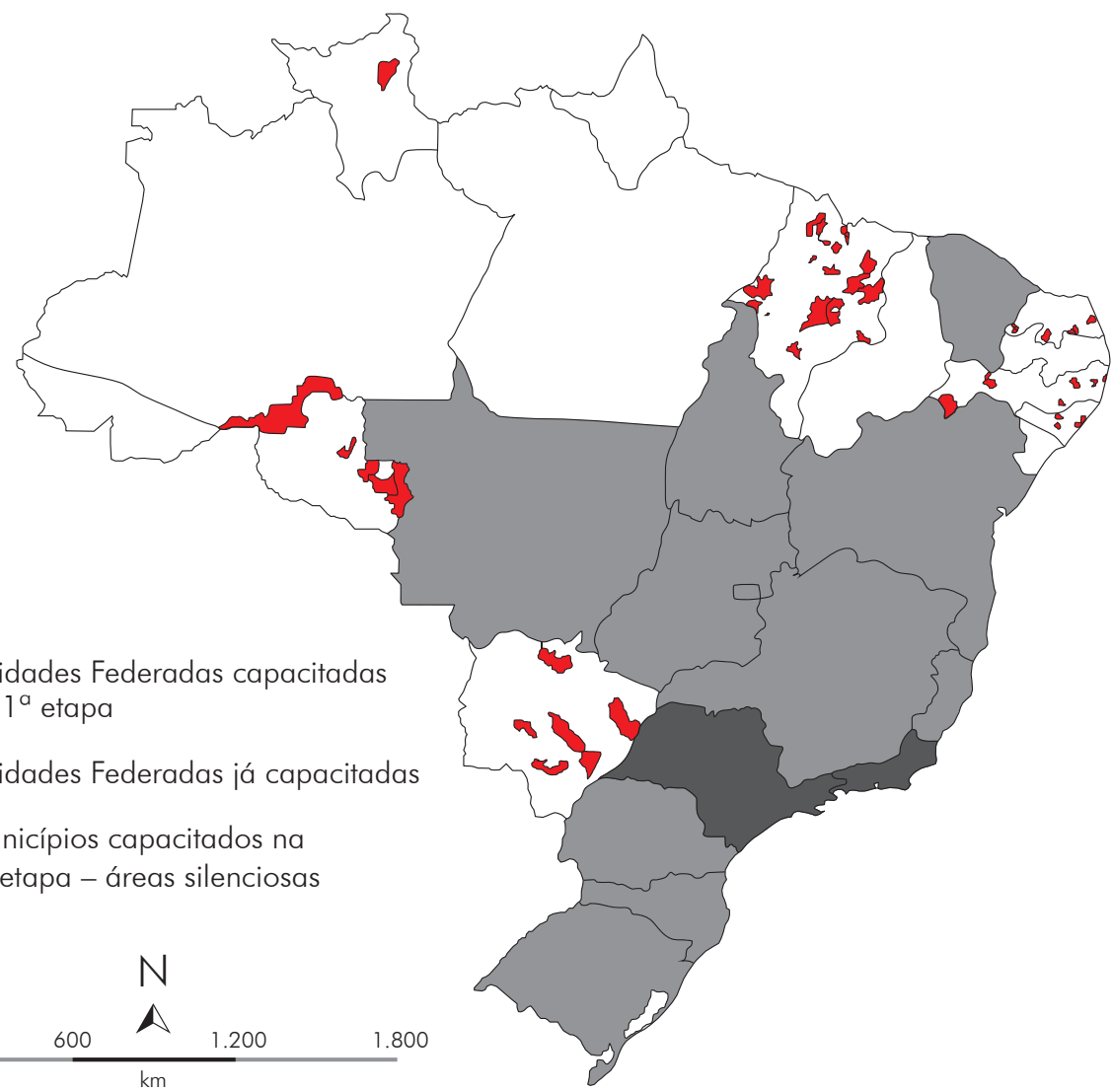

Figura 1 - Distribuição dos municípios brasileiros e das Unidades Federadas com profissionais capacitados para proposta de estruturação da Rede Nacional de Vigilância de Ambientes para Febre Maculosa e Outras Riquetsioses no Brasil 
Tabela 1 - Espécies de potenciais vetores coletados em áreas silenciosas do Brasil (2014/2015), durante o período de treinamento para vigilância de ambiente e investigação de casos para febre maculosa, segundo cada Unidade Federada capacitada

\begin{tabular}{|c|c|c|c|c|c|c|c|c|c|c|c|c|c|c|c|c|c|c|}
\hline \multirow{3}{*}{ Espécie de potencial vetor } & \multicolumn{18}{|c|}{ Unidades Federadas } \\
\hline & \multicolumn{3}{|c|}{ MS } & \multicolumn{3}{|c|}{ PE } & \multicolumn{3}{|c|}{$\mathrm{RO}$} & \multicolumn{3}{|c|}{ RR } & \multicolumn{3}{|c|}{ RN } & \multicolumn{3}{|c|}{ MA } \\
\hline & $N$ & Hosp & $\%$ & $N$ & Hosp & $\%$ & $\mathrm{~N}$ & Hosp & $\%$ & $\mathrm{~N}$ & Hosp & $\%$ & N & Hosp & $\%$ & $\mathrm{~N}$ & Hosp & $\%$ \\
\hline \multicolumn{19}{|l|}{ Carrapatos } \\
\hline Amblyomma cajennense & - & - & - & - & - & - & 22 & A & - & 13 & A & 50,0 & - & - & - & - & - & - \\
\hline Amblyomma naponense & - & - & - & - & - & - & 3 & A & - & - & - & - & - & - & - & - & - & - \\
\hline Amblyomma scalpturatum & - & - & - & - & - & - & 1 & A & - & - & - & - & - & - & - & - & - & - \\
\hline Amblyomma pseudoconcolor & - & - & - & 20 & T & 90,9 & - & - & - & - & - & - & - & - & - & - & - & - \\
\hline Dermacentor nitens & - & - & - & - & - & - & 177 & E & 0,8 & 63 & E & - & 176 & $E / B$ & - & 14 & E & 2,7 \\
\hline Rhipicephalus sanguineus & 108 & $\mathrm{~A} / \mathrm{C}$ & - & 23 & C & - & 117 & $\mathrm{~A} / \mathrm{C}$ & 1,5 & 238 & $\mathrm{~A} / \mathrm{C} / \mathrm{E}$ & 1,7 & 208 & $\mathrm{~A} / \mathrm{C} / \mathrm{B}$ & - & 183 & $\mathrm{~A} / \mathrm{C}$ & 2,7 \\
\hline Rhipicephalus microplus & 2 & B & - & - & - & - & 41 & $B / E$ & 9,0 & 10 & B & 16,6 & 72 & $E / B$ & 5,8 & - & - & - \\
\hline \multicolumn{19}{|l|}{ Pulgas } \\
\hline Ctenocephalides felis & 2 & G & - & - & - & - & 10 & C & 100,0 & 3 & C & - & 17 & C & - & - & - & - \\
\hline Xenopsylla cheopis & - & - & - & - & - & - & 2 & A & - & - & - & - & - & - & - & - & - & - \\
\hline \multicolumn{19}{|l|}{ Ácaro } \\
\hline Tur turki & - & - & - & - & - & - & 3 & $\mathrm{R}$ & - & - & - & - & - & - & - & - & - & - \\
\hline
\end{tabular}

N: número de espécimes coletados; Hosp: local de coleta (A - ambiente, B - boi, C - cão, E - equinos, G - gato, R - rato, T - tatu); \%: percentual de positividade para riquétsia. Sinal convencional utilizado: - Dado numérico igual a zero não resultante de arredondamento.

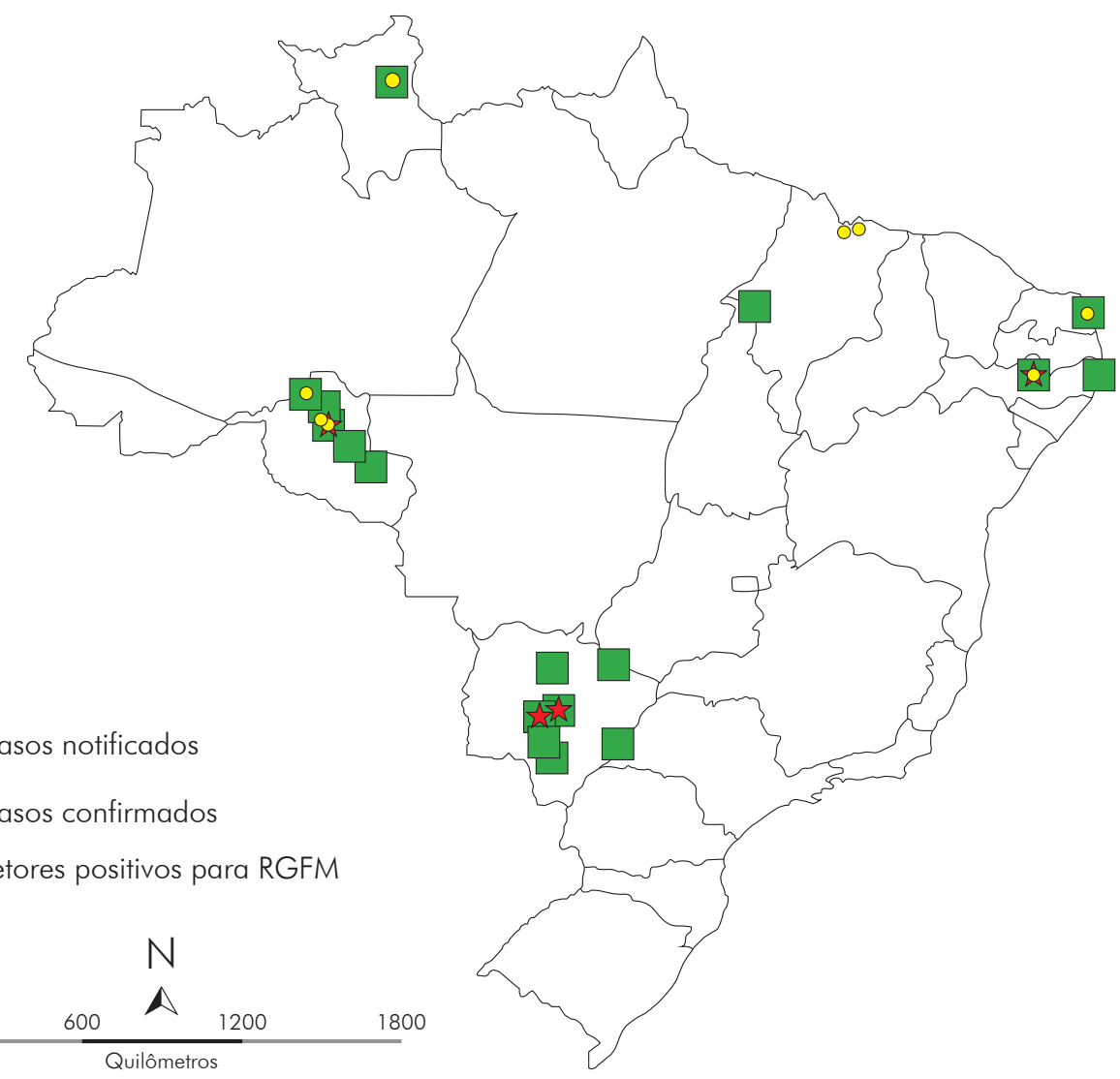

Figura 2 - Casos notificados e confirmados de febre maculosa, segundo o município de notificação e de infecção, e infecções por RGFM em vetores coletados em áreas silenciosas do Brasil, 2014 a 2015

\section{ANÁLISE DE DADOS EPIDEMIOLÓGICOS}

Foram registradas 40 notificações de casos suspeitos de febre maculosa nas áreas silenciosas investigadas no respectivo período. O MS concentrou o maior volume de notificações, $57,5 \%$ (23/40). Foram confirmados cinco casos de febre maculosa nos seguintes Estados: MS, 60\% (3/5); RO, 20\% (1/5); e PE, 20\% (1/5) (Figura 3). Nos Municípios de Campo Grande (2) e Dois Irmãos do Buriti em MS (1), Ariquemes em RO (1) e Sertânia em PE (1) houve confirmação de caso (Figura 2). 


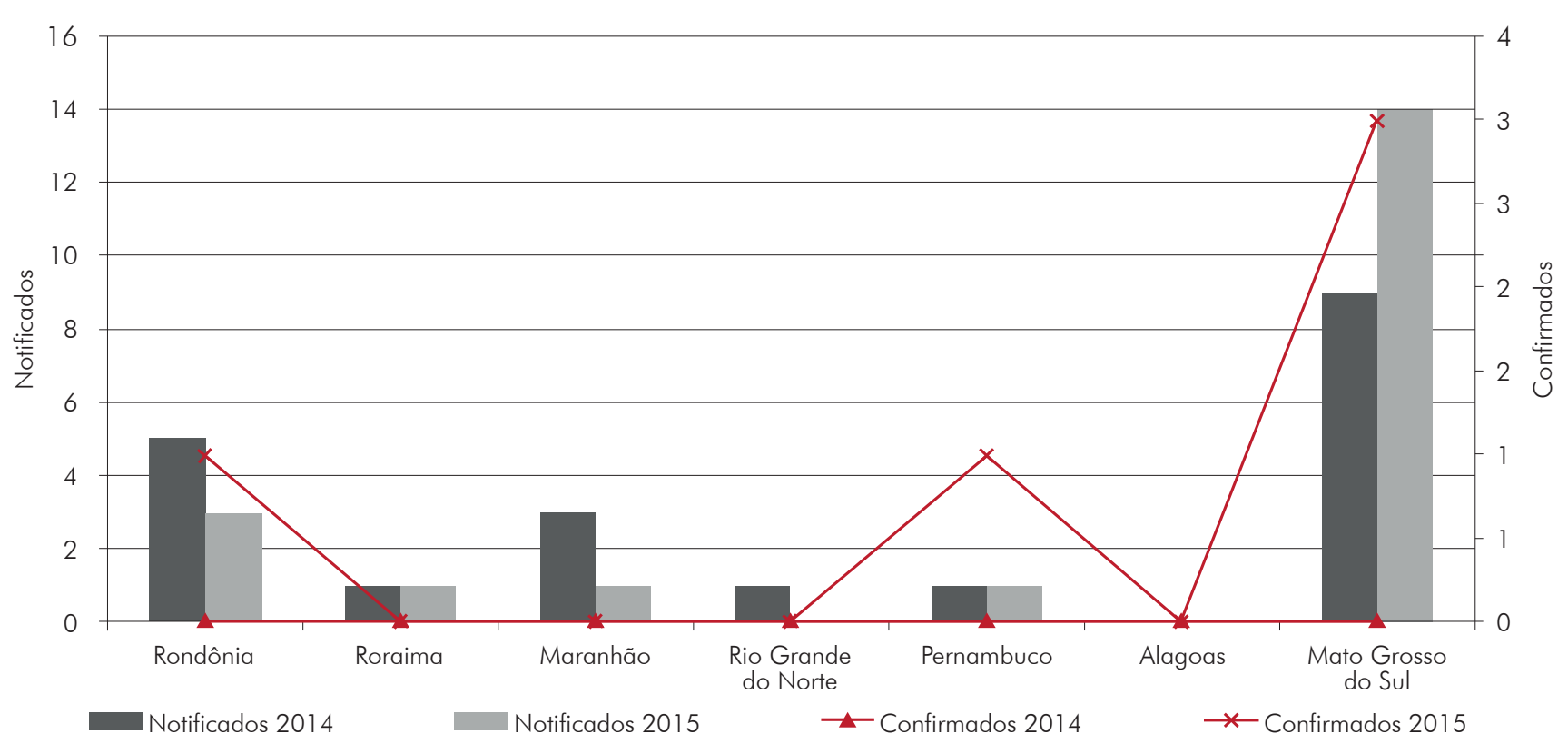

Figura 3 - Casos notificados e confirmados de febre maculosa, segundo as Unidades Federadas de notificação e da infecção, em áreas silenciosas do Brasil, 2014 a 2015

\section{DISCUSSÃO}

Com a iniciativa de intensificar a vigilância e explorar as áreas consideradas silenciosas para a presença da febre maculosa no Brasil, foi possível identificar a frequência de RGFM em $85 \%$ das Unidades Federadas que foram treinadas, sejam em vetores ou em humanos. No período em que ocorreram as intervenções, foram registrados os primeiros casos humanos de febre maculosa nos Estados de MS, $\mathrm{RO}$ e PE.

Os resultados das intervenções chamam a atenção para subnotificação de casos em áreas das Regiões Centro-Oeste, Norte e Nordeste do Brasil. Os casos de febre maculosa, mesmo em áreas reconhecidamente endêmicas, ocorrem em baixa incidência e com clínica inespecífica ${ }^{4,13}$, provavelmente esses são os principais fatores que dificultam o diagnóstico clínico em áreas onde a presença da doença ainda não foi assinalada. O exantema, principal sinal clínico da doença, nem sempre está presente ${ }^{11,12}$ e, em indivíduos de pele escura, quando presente, dificilmente é visualizado ${ }^{17}$.

Em áreas ermas do Brasil, o parasitismo por carrapatos é evento do cotidiano das populações, - que torna ainda mais difícil fazer o vínculo epidemiológico entre a exposição ao vetor e o desfecho (suspeita clínica da doença) 18,19,20. Aliado a esses fatores, existem parasitoses e arboviroses mais incidentes (malária, febre amarela, dengue, Zika e Chikungunya) que acometem as populações dessas áreas e que consomem quase toda a capacidade de resposta dos serviços de saúde dessas regiões ${ }^{21}$. Nesse sentido, a proposta de instituir a vigilância da febre maculosa em áreas silenciosas buscou otimizar atividades rotineiras de vigilância em saúde de outros programas, incluindo a febre maculosa entre o escopo das doenças sob a vigilância efetiva nessas áreas. Também foram discutidas formas de fazer uso oportuno da riqueza de informações biológicas (amostras) e ecoepidemiológicas, disponíveis de outros programas de vigilância em saúde e de outras instituições públicas, como currais municipais, abrigos de animais, centros de triagem de animais silvestres ou mesmo de instituições parceiras.

Recentemente, novas espécies de riquétsias foram identificadas em vetores no Brasil, como R. parkeri, Rickettsia sp. Mata Atlântica, Rickettsia amblyommii, Rickettsia andeanae e Rickettsia monteiroi3,8, muitas da quais a patogenicidade ainda é desconhecida. Por outro lado, algumas riquétsias transmitidas por carrapatos, como Rickettsia sp. Mata Atlântica, causam quadros clínicos mais brandos, o que poderia estar dificultando a captação destes registros e avaliação da real magnitude da febre maculosa em áreas silenciosas. Dessa forma, investigações que busquem caracterizar os agentes etiológicos que acometem as populações humanas nessas regiões são fundamentais para a compreensão da doença.

A amplitude geográfica e a vasta biodiversidade dessas regiões tornam estas áreas como campo prioritário nas investigações das potenciais doenças transmitidas por carrapatos. Igualmente, a caracterização molecular de RGFM é imprescindível para melhor entendimento do potencial patogênico das riquétsias detectadas em uma área, bem como para avaliar a vulnerabilidade para $\circ$ potencial estabelecimento do ciclo epidêmico ${ }^{22}$.

A positividade de RGFM, em vetores associados à investigação de casos humanos, chama atenção para um ciclo silvestre de riquétsias, cujo potencial patogênico ainda precisa ser esclarecido8,23. Também destaca-se a presença de RGFM em Rhipicephalus sanguineus e Ctenocephalides felis, vetores cujo potencial transmissor no Brasil ainda é dúbio, mas com evidências na manutenção de RGFM em ciclos enzoóticos ${ }^{24,25}$. 
processo da formação da Rede Nacional de Vigilância de Ambientes para Febre Maculosa e Outras Riquetsioses 9 tem possibilitado a oportunidade das intervenções, além da uniformização de métodos de investigação e vigilância epidemiológica (predição e prevenção) com frequente resposta na detecção de casos, visando a redução da morbimortalidade, objetivos prioritários do sistema de vigilância da febre maculosa no Brasil.

\section{CONCLUSÃO}

Conclui-se que a febre maculosa está presente em áreas consideradas silenciosas para a doença no Brasil. As intervenções, no âmbito da vigilância epidemiológica e ambiental, possibilitaram a detecção de riquétsias em vetores e a confirmação dos primeiros casos humanos da doença nos Estados do MS, RO e PE. Os resultados das intervenções realizadas no âmbito das qualificações técnicas mostraram a importância dessas iniciativas, tendo em vista que muitas doenças não são registradas pelo Sistema Único de Saúde devido ao desconhecimento da epidemiologia por parte dos profissionais de saúde. A febre maculosa causa doença grave na Região Sudeste do Brasil e, por isso, o conhecimento de sua distribuição em áreas consideradas silenciosas é importante para o desencadeamento de estratégias de prevenção e controle.

\section{AGRADECIMENTOS}

Às equipes técnicas das Secretarias Estaduais de Saúde dos Estados envolvidos, aos laboratórios centrais de saúde pública, laboratórios de entomologia, unidades de vigilância de zoonoses, universidades e à Empresa Brasileira de Pesquisa Agropecuária/MS.

\section{CONTRIBUIÇÃO DOS AUTORES}

Oliveira SV, Pereira SVC, Caldas EP e Gazeta GS contribuíram no planejamento da atividade; Fonseca LX, Pinna FV, Amorim M e Cardoso KM contribuíram no apoio técnico e operacional; Oliveira SV, Gazeta GS, Serra-Freire NM e Borsoi ABP contribuíram como monitores da atividade; e Oliveira SV, Caldas EP, Gazeta GS, na redação e revisão do manuscrito. Todos os autores aprovaram a versão final do artigo e são responsáveis por todos os aspectos do trabalho, incluindo a garantia de sua precisão e integridade.

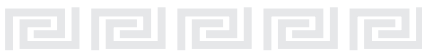

\section{Surveillance of environments of Rocky Mountain spotted fever: exploring the quiet areas of Brazil}

\section{ABSTRACT}

This study described the interventions in quiet areas of Brazil, from the aspect of health promotion. The areas were evaluated from 2014 to 2015, including the following Federal Units: Mato Grosso do Sul, Alagoas, Pernambuco, Rondônia, Roraima, Rio Grande do Norte and Maranhão. Data on surveillance spotted fever were evaluated by notification forms available in the Notifiable Diseases Information System (SINAN), as well as vectors and the spotted fever group rickettsial infections (SFGR). A total of 1,528 vectors were collected from ten different species, whose the highest diversity was observed in Rondônia. In this study, $85 \%$ of States which were investigated showed SFGR in vectors or human, and Mato Grosso do Sul, Rondônia and Pernambuco were the most prevalent ones. The results showed the necessity of ongoing training of health and environment professionals in order to improve data about the presence of Rocky Mountain spotted fever among us.

Keywords: Tick-Borne Diseases; Zoonoses; Rocky Mountain Spotted Fever; Inservice Training; Sentinel Surveillance.

\section{Vigilancia de ambientes de la fiebre maculosa: explorando las áreas silenciosas de Brasil}

\section{RESUMEN}

El presente estudio describió las intervenciones realizadas en áreas silenciosas de Brasil, bajo el aspecto de la promoción de la salud. Las áreas evaluadas durante el período de 2014 a 2015 comprendieron las siguientes Unidades Federadas: Mato Grosso do Sul, Alagoas, Pernambuco, Rondônia, Roraima, Rio Grande do Norte y Maranhão. Se evaluaron los datos sobre la vigilancia de la fiebre maculosa (o manchada) a través de las fichas epidemiológicas disponibles en el Sistema de Información de Agravamiento de Notificación (SINAN), bien como la fauna de vectores y de las infecciones por riquettsias del grupo de la fiebre maculosa (RGFM). Se recolectaron 1.528 vectores de diez especies diferentes, cuya mayor diversidad se observó en Rondônia. En este estudio, 85\% de los Estados investigados presentaron RGFM en vectores o en humanos, siendo los Estados más prevalentes Mato Grosso do Sul, Rondônia y Pernambuco. Los resultados presentados señalan la necesidad permanente de cualificación de los equipos de profesionales de salud y ambiente, con la finalidad de perfeccionar los datos sobre la fiebre maculosa en nuestro medio.

Palabras clave: Enfermedades Transmitidas por Garrapałas; Zoonosis; Fiebre Maculosa; Capacitación en Servicio; Vigilancia Centinela. 


\section{REFERÊNCIAS}

1 Parola P, Paddock CD, Raoult D. Tick-borne rickettsioses around the world: emerging diseases challenging old concepts. Clin Microbiol Rev. 2005 Oct;18(4):719-56.

2 Labruna MB. Ecology of rickettsia in South America. Ann N Y Acad Sci. 2009 May;1 166:156-66.

3 Parola P, Paddock CD, Socolovschi C, Labruna MB, Mediannikov $O$, Kernif $T$, et al. Update on tick-borne rickettsioses around the world: a geographic approach. Clin Microbiol Rev. 2013 Oct;26(4):657-702.

4 Barros e Silva PMR, Pereira SC, Fonseca LX, Maniglia FVP, Oliveira SV, Caldas EP. Febre maculosa: uma análise epidemiológica dos registros do sistema de vigilância do Brasil. Scient Plena. 2014;10(4):1-9.

5 Krawczak FS, Munõz-Leal S, Guztzazky AC, Oliveira SV, Santos FCP, Angerami RN, et al. Rickettsia sp. strain Atlantic rainforest infection in a patient from a spotted fever-endemic area in southern Brazil. Am J Trop Med Hyg. 2016 Sep;95(3):551-3.

6 Spolidorio MG, Labruna MB, Mantovani E, Brandão PE, Richtzenhain LJ, Yoshinari NH. Novel spotted fever group rickettsiosis, Brazil. Emerg Infect Dis. 2010 Mar;16(3):521-3.

7 Silva N, Eremeeva ME, Rozental T, Ribeiro GS, Paddock CD, Ramos EAG, et al. Eschar-associated spotted fever rickettsiosis, Bahia, Brazil. Emerg Infect Dis. 2011 Feb; 17(2):275-8.

8 Szabó MPJ, Pinter A, Labruna MB. Ecology, biology and distribution of spotted-fever tick vectors in Brazil. Front Cell Infect Microbiol. 2013 Jul;3:27.

9 Voizzoni VF, Silva AB, Cardoso KM, Santos FB, Stenzel B, Amorim M, et al. Genetic identification of Rickettsia sp. strain Atlantic rainforest in an endemic area of a mild spotted fever in Rio Grande do Sul state, Southern Brazil. Acta Trop. 2016 Oct;162:142-5.

10 Oliveira SV, Pereira SVC, Barros e Silva PMR, Pereira JM, Gomes V, Amorim M, et al. Vigilância de ambientes da febre maculosa brasileira e outras riquetsioses: a etapa inicial de uma proposta para a formação de rede. Rev Pan-Amaz Saude. 2015 set;6(3):67-71.

11 Ministério da Saúde do Brasil (BR). Secretaria de Vigilância em Saúde. Guia de vigilância em saúde: volume único. Brasília: Ministério da Saúde; 2014. Febre maculosa brasileira e outras riquetsioses; $p$. 445-55.

12 Ministério da Saúde (BR). Secretaria de Vigilância em Saúde. Doenças de "A a Z": febre maculosa, vídeo aulas. Brasília: Ministério da Saúde; 2015.
13 Oliveira SV, Guimarães JN, Reckziegel GC, Neves BMC, Araújo-Vilges KM, Fonseca LX, et al. An update on the epidemiological situation of spotted fever in Brazil. J Venom Anim Toxins Incl Trop Dis. $2016 ; 22(22): 1-8$.

14 Aragão H, Fonseca F. Notas de ixodologia: VIII. Lista e chave para os representantes da fauna ixodológica brasileira. Mem Inst Oswaldo Cruz. 1961 jul;59(2):115-30.

15 Azad AF, Webb L, Carl M, Dasch GA. Detection of rickettsiae in arthropod vectors by DNA amplification using the polymerase chain reaction. Ann N Y Acad Sci. 1990 Jun;590: 557-63.

16 Eremeeva M, Yu X, Raoult D. Differentiation among spotted fever group rickettsiae species by analysis of restriction fragment length polymorphism of PCR-amplified DNA. J Clin Microbiol. 1994 Mar;32(3):803-10.

17 Favacho ARM, Rozental T, Calic SB, Scofield MAM, Lemos ERS. Fatal Brazilian spotless fever caused by Rickettsia rickettsii in a dark-skinned patient. Rev Soc Bras Med Trop. 2011 May-Jun;44(3):395-6.

18 Lopes LB, Guterres A, Rozental T, Oliveira RC, Mares-Guia MA, Fernandes J, et al. Rickettsia bellii, Rickettsia amblyommii, and Laguna Negra hantavirus in an Indian reserve in the Brazilian Amazon. Parasit Vectors. 2014 Apr;7(191):1-7.

19 Ramos VN, Osava CF, Piovezan U, Szabó MPJ. Ticks on humans in the Pantanal wetlands, Brazil. Ticks Tick Borne Dis. 2014 Sep;5(5):497-9.

20 Szabó MP, Labruna MB, Castagnolli KC, Garcia MV, Pinter A, Veronez VA, et al. Ticks (Acari: Ixodidae) parasitizing humans in an Atlantic rainforest reserve of Southeastern Brazil with notes on host suitability. Exp Appl Acarol. 2006 Aug;39(3-4):339-46.

21 Rodrigues SG, Oliva OP, Araujo FAA, Martins LC, Chiang JO, Henriques DF, et al. Epidemiology of Saint Louis encephalitis virus in the Brazilian Amazon region and in the State of Mato Grosso do Sul, Brazil : elevated prevalence of antibodies in horses. Rev Pan-Amaz Saude. 2010 Mar;1 (1):81-6.

22 Spolidorio MG. Perfil sorológico e molecular de zoonoses transmitidas por carrapatos em humanos e animais domésticos oriundos de seis municípios do Estado do Espírito Santo [tese]. São Paulo (SP): Universidade de São Paulo, Faculdade de Medicina; 2009. 74 p.

23 Mclntosh D, Bezerra RA, Luz HR, Faccini JLH, Gaiotto FA, Giné GAF, et al. Detection of Rickettsia bellii and Rickettsia amblyommii in Amblyomma longirostre (Acari: Ixodidae) from Bahia state, Northeast Brazil. Braz J Microbiol. 2015 JulSep;46(3):879-83. 
24 Oliveira KA, Oliveira LS, Dias CCA, Silva Jr A, Almeida MR, Almada G, et al. Molecular identification of Rickettsia felis in ticks and fleas from an endemic area for Brazilian Spotted Fever. Mem Inst Oswaldo Cruz. 2008 Mar;103(2): $191-4$.
25 Rozental T, Favacho ARM, Barreira JD, Oliveira RC, Gomes R, Almeida DNP, et al. Rickettsia spp. infection in Rhipicephalus sanguineus ticks in a Brazilian spotted fever endemic rural area in Rio de Janeiro state, Brazil. Clin Microbiol Infect. 2009 Dec;15 Suppl 2:245-6. 